\title{
Adaptive Decode-and-Forward Cooperative Networks with Multiple Relay Nodes
}

\author{
Ha Nguyen $\mathrm{Vu} \cdot$ Hyung-Yun Kong
}

\begin{abstract}
We propose an adaptive cooperative scheme with a multi-relay node that achieves high bandwidth efficiency and achieves better SEP performance. In the proposed protocol, if the quality of the direct channel is better than that of the all channels from relays to destination, the source will transmit directly to the destination. Otherwise, the source broadcasts the signal and then a potential relay will be chosen to help the source. A re-transmission will also occur if the potential relay cannot be detected. The spectral efficiency is first derived by calculating the probability of each mode, i.e., direct and cooperation transmission. Subsequently, the SEP performance of M-PSK modulation for the scheme is analyzed by considering each event where the source transmits data to the destination. Finally, the obtained analytical results are verified through computer simulations.
\end{abstract}

Key words : Adaptive Cooperation, Sep Performance, Spectral Efficiency, Relay Selection.

\section{I . Introduction}

Cooperative diversity techniques have attracted considerable and increasing attention over the past several years [1] [3]. In cooperative communication, relays are assigned to help a source $(S)$ forward its information to a destination $(D)$; hence, a virtual antenna array can be formed, thereby achieving diversity. Various cooperative diversity protocols have been previously proposed and analyzed [1 4]. However, cooperation almost always occurs in these literature schemes. This may waste bandwidth and power allocated for the relaying strategy if the relay fails to decode the source's information or if the direct channel is inadequate for the destination detecting the data. Previous work by others [5] and also by our group [6], which investigated the adaptive cooperative scheme with one $R$, showed some benefits regarding the data rate and the reliability of transmission when compared with other published alternatives.

In this paper, we focus on the adaptive cooperation scheme with multiple relays in which the time and bandwidth can be saved, which has a higher reliability performance than conventional multi-relay schemes. First, by using the instantaneous SNR as the performance measure, $D$ checks which of the direct path or the relay path is better, and then chooses the path having the higher SNR for the transmission. If the direct channel is better than all other relay channels, then the source will directly transmit data to the destination with all power con- sumption. In contrast, if the direct link is not the best one, then the selection of the DF strategy will occur with the help of the relays that correctly decode the received signal. In this case, the source broadcasts the signal with a lower power in the first time slot. Afterward, the relay having the highest instantaneous SNR to the destination is chosen to retransmit the re-encoded signal. We also apply the re-transmission at $\mathrm{S}$ when a potential relay cannot be defined.

Through mathematical analysis, we have studied the proposed scheme by investigating the spectral efficiency and the symbol error probability (SEP) performance. We obtain the spectral efficiency by deriving the probability of each mode (i.e., direct transmission and two-phase transmission). Afterward, with the notation that the spectral efficiency of the two-phase mode equals one half of that of the direct transmission (DT) mode (i.e., one symbol is transmitted within one timeslot with DT mode and within two timeslots with two-phase mode), we can calculate the average efficiency of the scheme. Subsequently, we obtain the SEP performance of this scheme by considering two events where a potential relay that helps the communication between the source and the destination either exists or is absent. Finally, we use Monte-Carlo simulations to confirm the results of the mathematical analysis.

\section{II . Channel Model}

We consider a cooperative wireless network, as shown

Manuscript received July 6, 2010 ; revised November 22, 2010. (ID No. 20100706-023J)

Department of Electrical Engineering, University of Ulsan, Ulsan, Korea

Corresponding Author : Hyung Yun Kong (e-mail : hkong@mail.ulsan.ac.kr) 


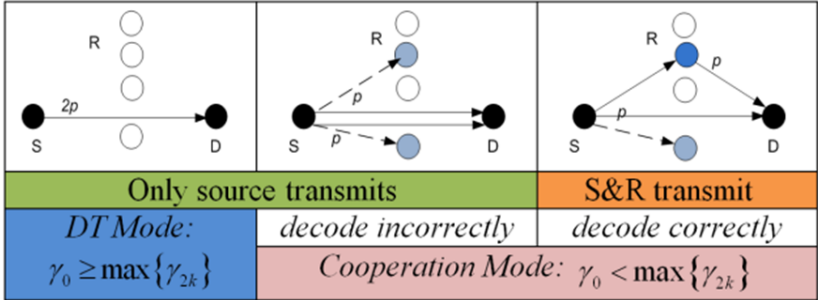

Fig. 1. Adaptive cooperative scheme with multi-relay.

in Fig. 1, where the source $(S)$ sends symbols to the destination $(D)$ with the assistance of $K$ DF relays $\left(R_{k}, k=1, \ldots, K\right)$. Each terminal is equipped with a single-antenna transceiver and operates in a half-duplex mode over frequency-flat block Rayleigh fading channels. We denote $\gamma_{0}, \gamma_{1 k}$, and $\gamma_{2 k}$ as $S-D, S-R_{k}$, and $R_{k}-D$ SNRs, which are modeled as exponential random variables with the means $\Omega_{0}^{-1}, \Omega_{1 k}^{-1}$, and $\Omega_{2 k}^{-1}$, respectively.

At the beginning of the transmission frame, $D$ collects and compares all of the channel coefficients between it and the other terminals, and then makes and broadcasts a K-bit message where, if the $i^{-t h}$ bit is 1 , it means that $\gamma_{0}<\gamma_{2 k}$ and vice versa. Therefore, based on this message, $S$ determines whether or not the event $\gamma_{0} \geq$ $\max \left\{\gamma_{2 k}\right\}$ happens. A group (C) of the active relays that have a channel to $D$ better than that of the $S-D$ channel can also be detected. For the case where the number of relays is not very large, the overhead for limited feedback information from the destination to the source is negligible.

If $\gamma_{0} \geq \max \left\{\gamma_{2 k}\right\} \quad\left(\Phi^{2}, 2\right.$-phase mode), $S$ employs direct transmission (DT) with total power consumption. Thus, the instantaneous SNR of the received signal at the destination can be given as:

$$
\gamma_{\Phi^{D}}=P \gamma_{0} / N_{0}
$$

where $P$ is the total transmitted power and $N_{0}$ is the power of the additive noise.

If $\gamma_{0}<\max \left\{\gamma_{2 k}\right\}$ ( $\Phi^{2}, 2$-phase mode), in the first time slot, $S$ broadcasts its symbol to $D$ and the active relays (in group (C)). In the next time slot, if all relays fail to decode the symbol, they will inform $S$ to transmit again with the remaining power. Here, we assume that the channels are unchanged in two time slots and the Maximum Ratio Combining (MRC) technique is applied at the destination. Thus, the end-to-end instantaneous SNR can be given as:

$$
\gamma_{\Phi^{2}}^{m c}=\left(P_{1} \gamma_{0}+P_{2} \gamma_{0}\right) / N_{0}=P \gamma_{0} / N_{0}
$$

where $P_{1}$ and $P_{2}$ present the transmitted powers of two time slots.

On the other hand, if some relays (at least one) decode the received signals correctly, the relay with the highest SNR to the destination is chosen to help the transmission from the source. Here, a previous relay selection strategy [3] can be employed to detect the best relay. In this case, the end-to-end instantaneous SNR can be modeled as:

$$
\gamma_{\Phi^{2}}^{c o}=\left(P_{1} \gamma_{0}+P_{2} \gamma_{R D}\right) / N_{0}
$$

where $g_{R D}$ denotes the instantaneous SNR from the chosen relay to $D$. In this letter, for the theoretical analysis, we assume that the relay can decide whether the symbol is decoded correctly or imperfectly. This assumption can be achieved in the real-time system by using Cyclic Redundancy Check code to check the signal.

\section{The Spectral Efficiency}

In this section, we obtain the probability of the direct-transmission and the relay-cooperation modes. Based on these probabilities, we then calculate the average spectral efficiency of this proposed scheme. Due to the exponential distribution of $\gamma_{0}$ 와 $\gamma_{2 k}$, the probability of the event that $\gamma_{2 k}<\gamma_{0}$ with a value of $\gamma_{0}$ is:

$$
\operatorname{Pr}\left(\gamma_{2 k}<\gamma_{0}\right)=\int_{0}^{\gamma_{0}} \Omega_{2 k} e^{-\Omega_{2 k} \gamma_{2 k}} d \gamma_{2 k}=1-e^{-\Omega_{2 k} \gamma_{0}}
$$

Therefore, for a certain value of $\gamma_{0}$, the probabilities of DT and 2-phase modes can be given respectively as:

$$
\begin{aligned}
& \operatorname{Pr}\left(\Phi^{D}, \gamma_{0}\right)=\operatorname{Pr}\left\{\max \left(\gamma_{2 k}\right)<\gamma_{0}\right\}=\prod_{k=1}^{K}\left(1-e^{-\Omega_{2 k} \gamma_{0}}\right) \\
& \operatorname{Pr}\left(\Phi^{2}, \gamma_{0}\right)=1-\prod_{k=1}^{K}\left(1-e^{-\Omega_{2 \gamma_{0} \gamma_{0}}}\right)=\sum_{i=1}^{K}(-1)^{i-1} \sum_{\substack{n_{1}, \ldots, n_{i}=1 \\
n_{1}<\ldots<n_{i}}}^{K} e^{-\beta_{\gamma} \gamma_{0}}
\end{aligned}
$$

where $\beta_{i}=\sum_{l=1}^{i} \Omega_{2 n_{l}}$ and $\sum_{\substack{n_{1}, \ldots, n_{i}=1 \\ n_{1}<\ldots<n_{i}}}^{K}()$ presents for the summation with the parameters $n_{1}, \ldots, n_{i}$ run from 1 to $K$ with the condition $n_{1}<\ldots<n_{i}$. By averaging the two equations above over $g(0)$, the probabilities of two modes can be achieved as follows:

$$
\begin{aligned}
\operatorname{Pr}\left(\Phi^{2}\right) & =\int_{0}^{\infty} \Omega_{0} e^{-\Omega_{0} \gamma_{0}} \sum_{i=1}^{K}(-1)^{i-1} \sum_{\substack{n_{1}, \ldots, n_{j}=1 \\
n_{1}<\ldots<n_{i}}}^{K} e^{-\beta_{i} \gamma_{0}} d \gamma_{0} \\
& =\sum_{i=1}^{K}(-1)^{i-1} \sum_{\substack{n_{1}, \ldots, n_{i}=1 \\
n_{1}<\ldots<n_{i}}}^{\Omega_{0}} \frac{\Omega_{0}}{\Omega_{0}+\beta_{i}} \\
\operatorname{Pr}\left(\Phi^{D}\right) & =1-\operatorname{Pr}\left(\Phi^{2}\right)=1-\sum_{i=1}^{K}(-1)^{i-1} \sum_{\substack{n_{1}, \ldots, n_{i}=1 \\
n_{1}<\ldots<n_{i}}}^{K} \frac{\Omega_{0}}{\Omega_{0}+\beta_{i}}
\end{aligned}
$$


Let $r$ denote the spectral efficiency of the DT mode, then the spectral efficiency of the 2-phase one is given as $r / 2$ (using two time slots). Hence, the expected spectral efficiency of our scheme can be defined as:

$$
\tilde{r}=\operatorname{Pr}\left(\Phi^{D}\right) r+\operatorname{Pr}\left(\Phi^{2}\right) \frac{r}{2}=\left[2+\sum_{i=1}^{K}(-1)^{i} \sum_{\substack{n_{1}, \ldots, n_{i}=1 \\ n_{1}<\ldots<n_{i}}}^{K} \frac{\Omega_{0}}{\Omega_{0}+\beta_{i}}\right] \frac{r}{2}
$$

\section{SEP Performance Analysis}

We consider two events: where only $S$ transmits signals $\left(E^{1}\right)$ and where there is one relay cooperating with $S$ to transmit signals to $D\left(E^{2}\right)$. The end-to-end SNR at $D$ of each event can be given as:

$$
\begin{aligned}
& \gamma_{E^{1}}=\gamma_{\Phi^{D}}=\gamma_{\Phi^{2}}^{\in c}=P \gamma_{0} / N_{0} \\
& \gamma_{E^{2}}=\gamma_{\Phi^{2}}^{\infty}=\left(P_{1} \gamma_{0}+P_{2} \gamma_{R D}\right) / N_{0}
\end{aligned}
$$

With two these divisions, the end-to-end SEP of this scheme can be written as:

$$
\begin{aligned}
P_{s}(\gamma) & =P\left(\gamma_{E^{1}} \mid E^{1}\right) \operatorname{Pr}\left(E^{1}\right)+P\left(\gamma_{E^{2}} \mid E^{2}\right) \operatorname{Pr}\left(E^{2}\right) \\
& =P_{E^{1}}+P_{E^{2}}
\end{aligned}
$$

where $P_{E^{1}}=P\left(\gamma_{E^{1}} \mid E^{1}\right) . P_{r}\left(E^{1}\right)$ denotes the SEP of the event where only the source transmits a signal to the destination and $P_{E^{2}}=P\left(\gamma_{E^{2}} \mid E^{2}\right) . P_{r}\left(E^{2}\right)$ presents the SEP of the second event.

\section{4-1 SEP of the First Event}

In this event, no relay satisfied the conditions to cooperate with $S$. This means that for $R_{k}, \gamma_{2 k} \leq \gamma_{0}$; or, if $\gamma_{2 k}>\gamma_{0}, R_{k}$ fails to decode the received signal. Therefore, the probability of the first event can be calculated as:

$$
\begin{array}{r}
\operatorname{Pr}\left(E^{1}, \gamma_{0}\right)=\prod_{k=1}^{K}\left\{\operatorname{Pr}\left(\gamma_{2 k}<\gamma_{0}\right)+\left[1-\operatorname{Pr}\left(\gamma_{2 k}<\gamma_{0}\right)\right] P_{S, k}\right\} \\
=\prod_{k=1}^{K}\left(1-e^{-\Omega_{22} \gamma_{0}}+e^{-\Omega_{22 \gamma_{0}}} P_{S, k}\right)=\prod_{k=1}^{K}\left(1-\overline{P_{S, k}} e^{-\Omega_{22 \gamma_{0}}}\right)
\end{array}
$$

where $\bar{u}=1-u$ and $P_{S, k}$ is the SEP of the channel from $\mathrm{S}$ to $R_{k}$. Here, we consider the M-PSK modulation; hence, $P_{S, k}$ can be given by using the alternative form of the Q-function, as:

$$
P_{S, k}=\frac{1}{\pi} \int_{0}^{a} \frac{\Omega_{1 k}}{\Omega_{1 k}+\frac{b P_{1}}{N_{0} \sin ^{2} \theta}} d \theta=B_{0}\left(1, \frac{b P_{1}}{\Omega_{1 k} N_{0}}\right)
$$

where $a=(M-1) \pi / M, b=\sin ^{2}(\pi / M)$ and

$$
B_{0}(x, y)=\frac{1}{\pi} \int_{0}^{a} \frac{\sin ^{2} \theta}{x \sin ^{2} \theta+y} d \theta
$$

From (10) and (13), the SEP of $E^{1}$ event can be achieved follow Appendix A-1 as:

$$
\begin{aligned}
& P_{E^{1}}=P\left(\gamma_{E^{\mid}} \mid E^{1}\right) \operatorname{Pr}\left(E^{1}\right) \\
& =\int_{0}^{\infty} P\left(\gamma_{E^{\mid}} \mid E^{1}, \gamma_{0}\right) \operatorname{Pr}\left(E^{1}, \gamma_{0}\right) f_{\gamma_{0}}\left(\gamma_{0}\right) d \gamma_{0} \\
& =B_{0}\left(1, \frac{b P}{\Omega_{0} N_{0}}\right)-\sum_{i=1}^{K}(-1)^{i-1} \sum_{\substack{n_{0}, \ldots, n_{i}=1 \\
n_{1}<\ldots, \ldots n_{i}}}^{K} A_{i} B_{0}\left(1+\frac{\beta_{i}}{\Omega_{0}}, \frac{b P}{\Omega_{0} N_{0}}\right)
\end{aligned}
$$

where $A_{i}=\prod_{l=1}^{i} \overline{P_{S, n_{l}}}$.

\section{4-2 SEP of the Second Event}

In this event, one relay cooperates with the source to transmit the signal to its destination. In order to calculate the SEP of this event, we first consider an occurrence where one relay is chosen and we determine the SEP of the scheme when that relay helps the transmission from the source to the destination. Then, by making a summation of all of the outcomes, we can obtain the expected result.

Clearly, $R_{i}$ is selected if and only if $\gamma_{2 i}>\gamma_{0}$, its received signal is decoded successfully and the other relay has the $R-D$ instantaneous SNR is smaller than $\gamma_{2 i}$ or cannot decode correctly if $R-D$ SNR is larger than $\gamma_{2 i}$ Therefore, the probability that $R_{i}$ is chosen can be given by:

$$
\operatorname{Pr}_{i}\left(\gamma_{0}\right)=\overline{P_{S, i}} \operatorname{Pr}\left(\gamma_{0}<\gamma_{2 i}\right) \prod_{k=1, \neq i}^{K}\left(1-\overline{P_{S, k}} e^{-\Omega_{2 k} \gamma_{2 i}}\right)
$$

From previous work $(10,16)$ and following the mathematical analysis in Appendix A-2, the SEP of this case can be achieved as:

$P_{E^{2}}^{i}=P^{i}\left(\gamma_{E^{2}} \mid E^{2}\right) \cdot \operatorname{Pr}_{i}\left(E^{2}\right)$

$=\int_{0}^{\infty} \int_{\gamma_{0}}^{\infty} P^{i}\left(\gamma_{E^{2}} \mid E^{2}, \gamma_{2 i}\right) \operatorname{Pr}_{i} f_{\gamma_{2}}\left(\gamma_{2 i}\right) d \gamma_{2 i} f_{\gamma_{0}}\left(\gamma_{0}\right) d \gamma_{0}$

$$
=\overline{P_{S, i}}\left[\begin{array}{l}
B_{1}\left(1, \frac{b P_{2}}{B_{0} \Omega_{2 i}}, 1+\frac{\Omega_{2 i}}{\Omega_{0}}, \frac{b P}{N_{0} \Omega_{0}}\right)-\sum_{k=1}^{K}(-1)^{k-1} \\
\sum_{\substack{n_{1}, \ldots, n_{k}=1 \\
n_{1}<}}^{K} A_{i k} B_{1}\left(1+\frac{\beta_{i k}}{\Omega_{2 i}}, \frac{b P_{2}}{N_{0} \Omega_{2 i}}, 1+\frac{\Omega_{2 i}+\beta_{i k}}{\Omega_{0}}, \frac{b P}{N_{0} \Omega_{0}}\right)
\end{array}\right]
$$

where $\beta_{i k}=\sum_{l=1}^{k} \Omega_{2 n_{l}}, A_{i k}=\prod_{l=1}^{k} \overline{P_{s, n_{l}}}$ and

$$
B_{1}(x, y, u, v)=\frac{1}{\pi} \int_{0}^{a} \frac{\sin ^{2} \theta}{x \sin ^{2} \theta+y} \frac{\sin ^{2} \theta}{u \sin ^{2} \theta+v} d \theta
$$

By making the summation for all the relays, we get the SEP of the second event as follows:

$$
P_{E^{2}}=\sum_{i=1}^{K} P_{E^{2}}^{i}
$$

Finally, the end-to-end SEP of the scheme can be 
achieved as:

$$
P_{s}(\gamma)=P_{E^{1}}+P_{E^{2}}=P_{E^{1}}+\sum_{i=1}^{K} P_{E^{2}}^{i}
$$

\section{Simulation Result}

In this section, we use a Monte-Carlo simulation to evaluate the performance of the proposed cooperation schemes in terms of their spectral efficiency and bit error probabilities for the case where an equal power allocation is used, $P_{1}=P_{2}=P / 2$.

Fig. 2 depicts the spectral efficiency of the adaptive and conventional cooperation schemes for different numbers of relays. We plot both simulation results and theoretical analysis given by eqn. (9) over the independent and identically distributed (i.i.d) and also not identically distributed (i.n.d) channel, with $r=1$ for the i.i.d case and the i.n.d cases, as in the figure. The spectral efficiency of the proposed scheme decreases to $1 / 2$ as $K$ rises. The explanation is that increasing the number of relays boosts the probability of $\gamma_{0}<\max \left\{\gamma_{2 k}\right\}$ occurring. In addition, the spectral efficiency of the conventional cooperative schemes, $R_{\text {conv }}=1 /(K+1)$ SPCU, is plotted to show the significant increase in spectral efficiency of the proposed scenario over conventional ones.

Fig. 3 illustrates the Symbol error probability with the M-PSK modulation $(M=2,4,8,16)$ versus $P / N_{0}$ of the proposed scheme with different numbers of relays $(\mathrm{K}=$ $1,2,3)$ in a unity channel-variance case $\Omega_{0}=\Omega_{1 k}=$ $\Omega_{2 k}=1$.

We can see that the scheme performs better as the number of relays increases, which can be explained based on the increase in the network's diversity. When a higher modulation level is used, a higher symbol error rate is achieved because of the increasing number of bits in

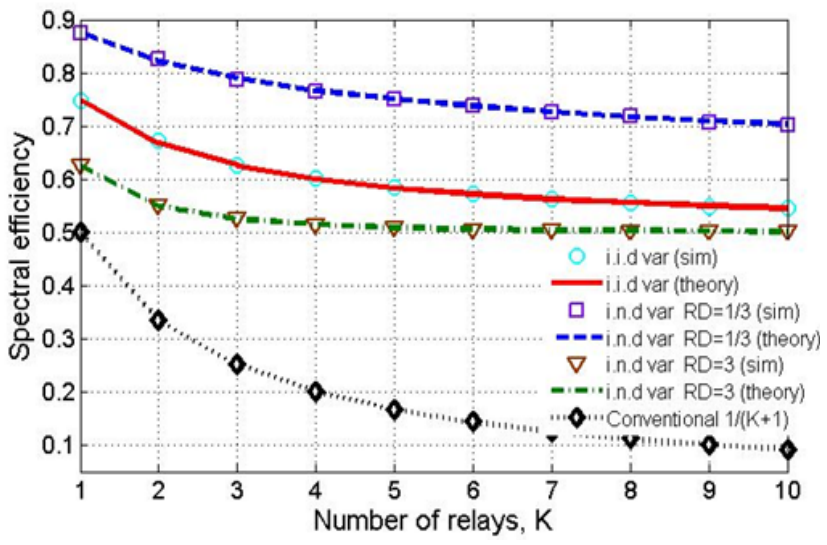

Fig. 2. Spectral efficiency versus the number of the relays $(K)$.

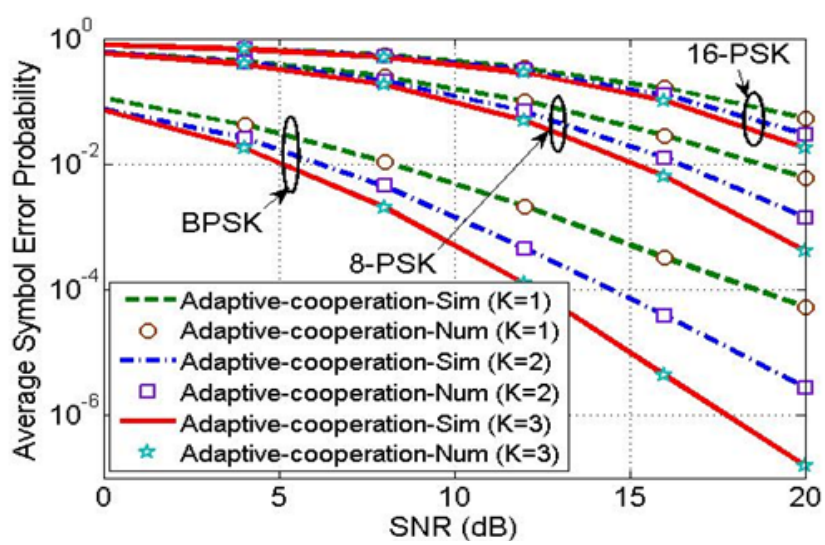

Fig. 3. Symbol-error-probability of the adaptive cooperative scheme with a different number of relays $K=1,2,3$.

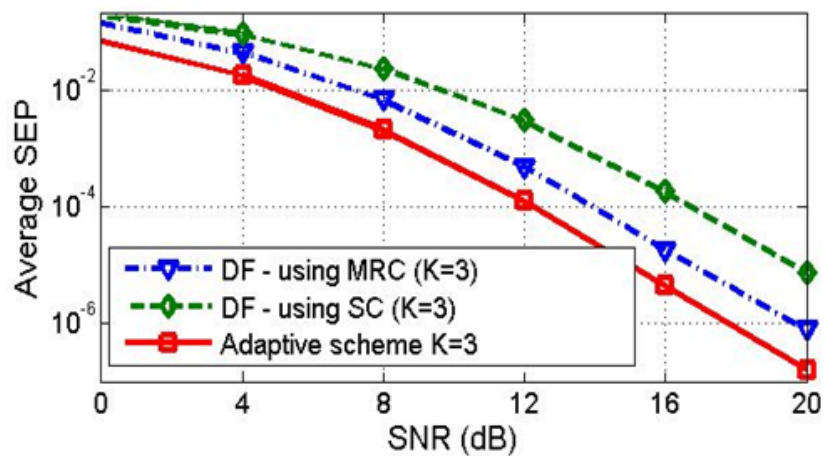

Fig. 4. Comparison of the average SEP of BPSK among three schemes: MRC, SC and adaptive scheme.

a codeword.

In the Fig. 4, the SEP performances of the BPSK modulation of three schemes (Decode and Forward multi-relay schemes with MRC [4] and SC diversity combining techniques at the destination) and that of the proposed scheme are plotted with the number of relays set at $3(K=3)$. According to this figure, we can see that, besides achieving higher spectral efficiency, as in Fig. 3, the SEP performance of our proposed scheme is superior to the conventional scenarios. In the comparison, our scheme outperforms the $\mathrm{SC}$ scheme by about $5 \mathrm{~dB}$ and the MRC scheme by $2 \mathrm{~dB}$ for all values of $P / N_{0}$.

Finally, we can see that all of the simulations and numerical results exactly match, which again confirms the good derivations of the analysis in this paper. The higher spectral efficiency and the lower transmission error rate compared to conventional schemes also indicate that our proposed scheme can perform as a potential transmission network in the future.

\section{Conclusion}

We considered the adaptive cooperation with relay-se- 
lection for the scheme multi-relay nodes in which the DF protocols can be applied whenever the direct link is of low quality. By applying the relay-selection technique, we reduce the number of transmissions, thereby saving time slots and bandwidth. In addition, the use of the adaptive protocol to choose between the DT and Decoded and Forward cooperative transmission allows our scheme to outperform some conventional methods.

\section{Appendices}

A-1 Calculate the SEP of the first case $\left(E^{1}\right)$ : the SEP for the first case can be given as follows:

$$
\begin{aligned}
& P_{E^{1}}=\int_{0}^{\infty} P\left(\gamma_{E^{1}} \mid E^{1}, \gamma_{0}\right) \operatorname{Pr}\left(E^{1}, \gamma_{0}\right) f_{\gamma_{0}}\left(\gamma_{0}\right) d \gamma_{0}
\end{aligned}
$$

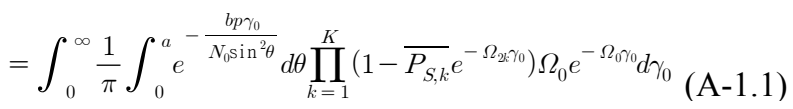

$$
\begin{aligned}
& P_{E^{1}}=\int_{0}^{\infty} \frac{1}{\pi} \int_{0}^{a} e^{-\frac{b P \gamma_{0}}{N_{0} \sin ^{2} \theta}} d \theta \\
& \times\left[1-\sum_{i=1}^{K}(-1)^{i-1} \sum_{\substack{n_{1}, n_{2}, \ldots, n_{i}=1 \\
n_{1}<n_{2}<\ldots<n_{i}}}^{K} A_{i} e^{-\beta \gamma_{0}}\right] \Omega_{0} e^{-\Omega_{0, \gamma_{0}}} d \gamma_{0} \\
& =\frac{1}{\pi} \int_{0}^{a} \frac{\Omega_{0}}{\Omega_{0}+\frac{b P}{N_{0} \sin ^{2} \theta}} d \theta \\
& -\sum_{i=1}^{K}(-1)^{i-1} \sum_{\substack{n_{1}, n_{2}, \ldots, n_{i}=1 \\
n_{1}<n_{2}<\ldots<n_{i}}}^{K} A_{i} B_{0}\left(1+\frac{\beta_{i}}{\Omega_{0}}, \frac{b P}{\Omega_{0} N_{0}}\right)
\end{aligned}
$$

where $\frac{1}{\pi} \int_{0}^{a} \exp \left(-b P \gamma_{0} / N_{0} \sin ^{2} \theta\right) d \theta$ presents for the SEP of M-PSK modulation and $\prod_{k=1}^{K}\left(1-\overline{P_{S, k}} e^{-\Omega_{2 k} \gamma_{0}}\right)$ is the probability of $E^{1}$ as a certain value of $\gamma_{0}$. By calculating this integral formula, the SEP of the first case is achieved as:

A-2 Calculate the SEP of the second case $\left(E^{2}\right)$ : when the relay $i^{\text {th }}$ is chosen to forward the signal to the destination, the SEP of the scheme can be given as follows:

$$
\begin{aligned}
P_{E^{2}}^{i}= & \int_{0}^{\infty} \int_{\gamma_{0}}^{\infty} P_{S}^{i}\left(\gamma_{E 2} \mid E^{2}, \gamma_{2 i}\right) \operatorname{Pr}_{i} f_{\gamma_{2}}\left(\gamma_{2 i}\right) d \gamma_{2 i} f_{\gamma_{0}}\left(\gamma_{0}\right) d \gamma_{0} \\
= & \int_{0}^{\infty} \int_{\gamma_{0}}^{\infty} \int_{0}^{a} \frac{e^{-\frac{b P_{1} \gamma_{0}+b P_{\gamma_{2}}}{N_{0} \sin ^{2} \theta}} d \theta}{\pi} \overline{P_{e, i}} \\
& \times \prod_{k=1, \neq i}^{K}\left(1-\overline{P_{e, k}} e^{-\Omega_{22 \gamma_{2} i}}\right) f_{\gamma 2 i}\left(\gamma_{2 i}\right) d \gamma_{2 i} f_{\gamma_{0}}\left(\gamma_{0}\right) d \gamma_{0}
\end{aligned}
$$

where $\frac{1}{\pi} \int_{0}^{a} \exp \left(-\left(b P_{1} \gamma_{0}+b P_{2} \gamma_{2}\right) / N_{0} \sin ^{2} \theta\right)$ presents for the SEP of M-PSK modulation and $\prod_{k=1, \neq i}^{K}(1-$
$\left.\overline{P_{e, k}} e^{-\Omega_{2 k} \gamma_{2 i}}\right) f_{\gamma_{2 i}}\left(\gamma_{2 i}\right)$ is the probability that the relay $i$ is chosen. By calculating this integral formula, the SEP of the second case, when the $i^{\text {th }}$ relay is chosen, can be achieved as:

$$
\begin{aligned}
& =\frac{\overline{P_{e, i}}}{\pi} \int_{0}^{a} \int_{0}^{\infty} \int_{\gamma_{0}}^{\infty}\left[1-\sum_{k=1}^{k-1}(-1)^{k} \sum_{\substack{n_{1}, n_{2}, \ldots, n_{k}=1, \neq i \\
n_{1}<n_{2}<\ldots<n_{k}}}^{K} A_{i k} e^{-\beta_{k i \gamma_{2}}}\right] \\
& =\frac{\overline{P_{e, i}}}{\pi} \int_{0}^{-\frac{b P_{1 \gamma_{0}}+b P_{2} \gamma_{2 i}}{N_{0} \sin ^{2} \theta}}\left[\begin{array}{l}
\Omega_{2 i} e^{-\Omega_{22} \gamma_{2}} d \gamma_{2 i} \Omega_{0} e^{-\Omega_{0 \gamma} \gamma_{0}} d \gamma_{0} d \theta \\
\frac{\Omega_{2 i}}{\Omega_{2 i}+\frac{b P_{2}}{N_{0} \sin ^{2} \theta}} \frac{\Omega_{0}}{\Omega_{0}+\Omega_{2 i}+\frac{b P}{N_{0} \sin ^{2} \theta}} \\
-\sum_{k=1}^{K-1}(-1)^{k} \sum_{n_{1}, n_{2}}
\end{array}\right.
\end{aligned}
$$

This research was supported by Basic Science Research Program through the National Research Foundation of Korea (NRF) funded by the Ministry of Education, Science and Technology(No. 2010-001 6782).

\section{References}

[1] A. Sendonaris, E. Erkip, and B. Aazhang, "User cooperation diversity - part i: System description," IEEE Transactions on Communications, vol. 51, no. 11, pp. 1927-1938, Nov. 2003.

[2] J. N. Laneman, D. N. C. Tse, and G. W. Wornell, "Cooperative diversity in wireless networks: Efficient protocols and outage behavior," IEEE Transactions on Information Theory, vol. 50, no. 12, pp. 3062-3080, 2004.

[3] A. Bletsas, A. Khisti, D. P. Reed, and A. Lippman, "A simple cooperative diversity method based on network path selection," IEEE Journal on Select Areas in Communications, vol. 24, no. 3, pp. 659-672, Mar. 2006.

[4] I. H. Lee, D. Kim, "Ber analysis for decode-and-forward relaying in dissimilar rayleigh fading channels," IEEE Communications Letters, vol. 11, no. 1, pp. 52-54, 2007.

[5] A. S. Ibrahim, A. K. Sadek, S. Weifeng, and K. J. R. Liu, "Cooperative communications with partial channel state information: When to cooperate?," in Global Telecommunications Conference, 2005. GLOBECOM '05. IEEE, pp. 3068-3072, 2005.

[6] H. N. Vu, H. Y. Kong, "Adaptive decode-and-forward cooperative networks with limited feedback," IEICE Communication Letter, vol. E93.B, no. 2, pp. 4-7, 2010. 


\section{Ha Nguyen $\mathrm{Vu}$}

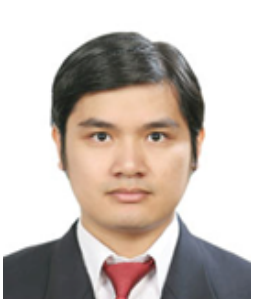

received a B.E. degree in electronics and telecommunications engineering from Ho Chi Minh City University of Technology, Vietnam, in 2007. He is currently working toward a Ph.D. degree in the Department of Electrical Engineering, University of Ulsan, Korea. His major research interests are combing techniques, OFDM transmission, cooperative communications, and Cognitive Radio networks.

\section{Hyung-Yun Kong}

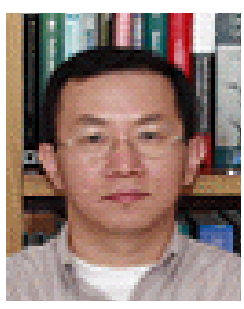

received M.E. and Ph.D. degrees in electrical engineering from Polytechnic University, Brooklyn, New York, USA, in 1991 and 1996, respectively, He received a $\mathrm{BE}$ in electrical engineering from the New York Institute of Technology, New York, in 1989. Since 1996, he has been employed by LG Electronics Co., Ltd., in the multimedia research lab, He received M.E. and Ph.D. degrees in electrical engineering from Polytechnic University, Brooklyn, New York, USA, in 1991 and 1996, respectively, He received a BE in electrical engineering from the New York Institute of Technology, New York, in 1989. Since 1996, he has been employed by LG Electronics Co., Ltd., in the multimedia research lab. Currently he is a professor in Electrical Engineering at University of Ulsan, Korea. His research area includes channel coding detection and estimation, cooperative communications, cognitive radio and sensor networks. He performs several projects supported by NRF (National Reserch foundation of Korea), Hyundai heavy industries Co. Ltd. 